\title{
All in the barn: who oversees agricultural research animals?
}

$\mathrm{V}$

ery few researchers require llamas for their studies, but Dr. Helen Zymansky had such a need. Zymansky, a professor at Great Eastern University's College of Agriculture, used small llama-derived antibodies (nanobodies) as part of her investigations on bovine immunodeficiency virus (BIV), a lentivirus with an uncertain impact on animal health, that is found in cattle from the U.S. and other countries. The research was funded by federal government grants and focused on determining if BIV affected the reproductive efficiency of dairy cows. The IACUC was aware of Zymansky's work because the llamas were housed in the same barn with animals used for studies overseen by the IACUC. However, because Zymansky's animals were being used to study the effect of BIV on reproductive efficiency of dairy cows, the research committee of the College of Agriculture, not the IACUC, approved and monitored Zymansky's work.

During an AAALAC site visit for the colleges of medicine and veterinary medicine, the visitation team went to the barn that housed Zymansky's animals and those under the jurisdiction of the IACUC. The visitors saw a llama with a generalized skin infection that was rubbing itself against the side of its stall. There was no record of any veterinary examination of the animal and no indication that the infection was being treated. The barn manager said that he had not noticed the problem. At the site visit exit briefing the visitors questioned the lack of an IACUC protocol for Zymansky's work and stated that the untreated infection will lead to a recommendation that AAALAC issue a mandatory notice indicating a need for more thorough animal monitoring and veterinary oversight. The schools replied that research on and for the benefit of agricultural animals did not fall under the jurisdiction of the IACUC and therefore no IACUC protocol was required, but they would inform the College of Agriculture research committee that the animal required medical care and the veterinarians would immediately contact Dr. Zymansky. The site visitors said they agreed with the immediate action to be taken. They then cited the
Guide for the Care and Use of Laboratory Animals ${ }^{1}$, which states that "Regardless of the category of research [i.e., agricultural or biomedical], institutions are expected to provide oversight of all research animals and ensure that pain and distress are minimized."

Did the schools of medicine and veterinary medicine respond appropriately in this situation? Do you think that the BIV study was biomedical or agricultural? Did the site visitors overstep their authority by recommending a mandatory item for correction when the IACUC claimed that it had no jurisdiction over the study?

\section{Jerald Silverman \\ University of Massachusetts Medical School, Worcester, MA, USA. \\ e-mail: Jerald.Silverman@umassmed.edu}

Published online: 22 January 2019

https://doi.org/10.1038/s41684-018-0221-6 References
1. Institute for Laboratory Animal Research. Guide for the Care and
Use of Laboratory Animals. 8th Ed., 33.(National Academies Press, Washington, DC, 2011)..

\section{Who's responsible for the llama drama?}

resis his scenario addresses IACUC oversight and responsibilities as they pertain to agricultural animal research. After a llama is noted to have an untreated generalized skin infection during the AAALAC site visit of the schools of medicine and veterinary medicine at Great Eastern University (GEU), the site visitors recommended the schools receive a mandatory notice "indicating the need for more thorough animal monitoring and veterinary oversight." The IACUC responded that since the llamas are part of a research study aimed at improving the reproductive efficiency of dairy cows, this research would not fall under its purview but that they would inform the College of Agriculture, whose research committee approved the work, and the PI of the need for veterinary care. Despite their assertion that research on and for the benefit of agricultural animals does not fall under the jurisdiction of the IACUC, their response to AAALAC's mandatory notice could have been more robust.

Assuming that GEU holds a PHS Assurance, OLAW encourages institutions to perform program oversight institutionwide using uniform and consistent standards for animal care and use regardless of funding source ${ }^{1}$. PHS Policy requires adherence to the Guide for the Care and Use of Laboratory Animals (Guide) which “... applies to agricultural animals...including those maintained in typical farm settings ${ }^{2}$." The Guide affirms that the species involved in research does not affect the fundamental roles and responsibilities of the institutional entities charged with regulatory oversight and gives IACUCs flexibility to categorize "research uses of agricultural animals and define standards for their care and use... based on both the researcher's goals and concerns for animal well-being ${ }^{3}$." As such, GEU's IACUC has jurisdiction over these animals despite this being agricultural research. In this case, the IACUC could ensure appropriate oversight by requesting that Dr. Zymansky provide documentation to the IACUC administrator and the Attending Veterinarian (AV) detailing the study goals, procedures (e.g. frequency of blood collection), and the qualified personnel providing the daily husbandry and veterinary care. Ideally, this information can be captured in a protocol and the IACUC can decide whether the protocol will adhere to the standards outlined in the Guide or the Guide for the Care and Use of Agricultural Animals in Research and Teaching (Ag Guide). In either case, veterinary care of these animals should be specifically outlined in their AAALAC Program Description. If the decision is made to adhere to the performance standards of the $\mathrm{Ag}$ Guide, then the IACUC will need to ensure that it is properly constituted and may need to add a member with agricultural animal experience. 